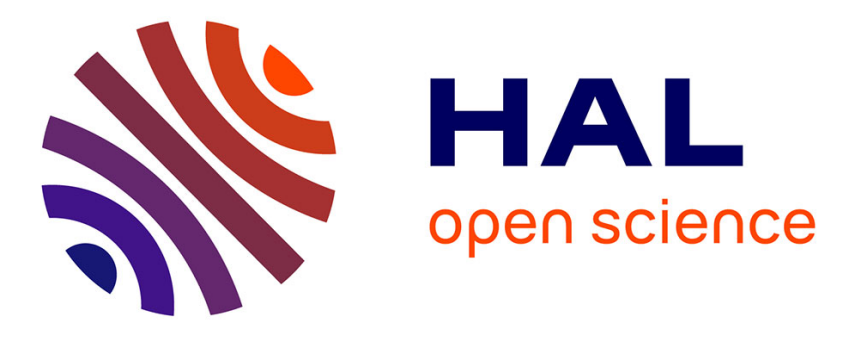

\title{
Tracking photoluminescent carbon nanomaterials in biological systems
}

Simon Haziza, Laurent Cognet, François Treussart

\section{To cite this version:}

Simon Haziza, Laurent Cognet, François Treussart. Tracking photoluminescent carbon nanomaterials in biological systems. Carbon Nanomaterials for Bioimaging, Bioanalysis, and Therapy, John Wiley \& Sons Ltd, pp.115-137, 2018, 10.1002/9781119373476.ch6 . hal-03133614

\section{HAL Id: hal-03133614 https://hal.science/hal-03133614}

Submitted on 6 Feb 2021

HAL is a multi-disciplinary open access archive for the deposit and dissemination of scientific research documents, whether they are published or not. The documents may come from teaching and research institutions in France or abroad, or from public or private research centers.
L'archive ouverte pluridisciplinaire HAL, est destinée au dépôt et à la diffusion de documents scientifiques de niveau recherche, publiés ou non, émanant des établissements d'enseignement et de recherche français ou étrangers, des laboratoires publics ou privés. 


\title{
Tracking photoluminescent carbon nanomaterials in biological systems
}

\author{
Simon Haziza ${ }^{1,2}$, Laurent Cognet $^{3,4}$ and François Treussart ${ }^{5}$ \\ ${ }^{1}$ James H. Clark Center for Biomedical Engineering \& Sciences, Stanford University, Stanford, CA \\ 94305, USA. \\ ${ }^{2}$ CNC Program, Stanford University, Stanford, CA 94305, USA \\ ${ }^{3}$ Univ. Bordeaux, Laboratoire Photonique Numérique et Nanosciences, UMR 5298, F-33400 Talence, \\ France \\ ${ }^{4}$ Institut d'Optique \& CNRS, LP2N UMR 5298, F-33400 Talence, France. \\ ${ }^{5}$ Laboratoire Aimé Cotton, CNRS, Univ. Paris-Sud, ENS Paris-Saclay and Univ. Paris-Saclay, F-91405 \\ Orsay, France
}

\section{Chapter summary}

This chapter highlights some recently published outstanding researches that have pushed further the boundary of photoluminescent carbon nanomaterials applications to biological systems and have brought invaluable insights into cellular and organism dynamics. From our perspective, there is a need for nanoparticle-based methodologies that allow researcher to access the spatiotemporal dynamics inherent to a wide variety of biological processes.

Two carbon allotropes have recently shown remarkable advances in neurosciences: fluorescent nanodiamonds (FND) (1) and single wall carbon nanotube (SWCNT) (2). We highlight in this chapter three examples of recent archetypical achievements based on the tracking of FNDs or SWCNTs while being aware that we are not exhaustive. Single particle tracking of FND was used to monitor the endosomal transport inside hippocampal neurons dissociated from mouse embryos and the author used this nanoparticle-based technique to unravel transport defects in mouse models of brain diseases. Single particle tracking of SWCNT was used to study the nanoscale organization and rheological properties of the extracellular space in acute slices of mouse brain. Altogether, this chapter reviews a decade of research related to FND and SWCNT tracking in cells (such as cancer cell lines and neurons) and organisms (such as zebrafish, drosophila embryos, C-elegans and mice).

\section{Introduction}


Many carbon allotropes nanoparticles are currently used in biology and biomedical applications, including fluorescent diamond nanocrystals, single-wall carbon nanotubes, carbon dots, reduced graphene oxide and fullerenes. Each of them have often specific applications. For example, fullerenes are mostly used for drug delivery in tumor therapy and for theranostic applications (3) whereas reduced graphene oxide are mainly used as a biomolecule sensor owing to its ability to strongly quench dye fluorescence (4). Carbon dots was used for cellular imaging via passive internalization in cell lines, although no appealing dynamic biological experiments were conducted (5). The two carbon nanomaterial allotropes that show great potentials are FND and SWCNT. They were shown to be used individually (like in extracellular or intracellular single particle tracking) or in cluster (like in tracking cellular system). This chapter starts with tracking of cells using FND, followed by a section of single particle tracking of FND inside living organism and inside living cancer cells and neurons from mouse embryo. The last section deals with the use of SWCNT for the study of the extracellular space nanoscale dimensions and local viscosity in a live brain tissue.

\section{Tracking cells in organisms with fluorescent nanodiamonds}

Many studies have shown that acid-treated high-pressure high-temperature (HPHT) synthesized $100 \mathrm{~nm}$-sized (and smaller) nanodiamonds (ND) can be efficiently internalized inside living cells and organisms. This spontaneous uptake happens in diverse cell types, including cancer cells (6) and primary neurons (1), following the common pathway of receptor mediated endocytosis (7). NDs are then trapped in endocytotic compartments at different stages of their evolution, down to the Iysosomes $(1,7)$. ND cellular internalization has very distinct characteristics, with (i) almost no exocytosis even during cell proliferation (8), and (ii) a very low induced toxicity on a few days scale as assessed from cell survival, morphogenesis (9), functions (10) and genotoxicity (11) measurements.

ND can be made fluorescent in the near infrared spectral region (600-750 $\mathrm{nm}$ wavelength range) by creating a nitrogen-vacancy (NV) color center within the diamond lattice, which requires the association of naturally present nitrogen $(\mathrm{N})$ impurities ( $\approx 100 \mathrm{ppm}$ abundance in HPHT diamond) with 
vacancy (V) induced by high energy particle irradiation $(12,13)$. NV-centers in diamond have remarkable properties (14): (i) a perfect photostability (no bleaching and no blinking) and (ii) an optically detectable magnetic resonance (ODMR) of the electronic spin of its negatively charged form $\left(\mathrm{NV}^{-}\right)$. The latter offers the possibility to modulate the fluorescence signal of FNDs with a variable external magnetic field (15). Furthermore, when NV centers are embedded in diamond nanoparticles of size much smaller than the optical excitation wavelength, their radiative lifetime substantially increases by dielectric screening effect (16). For example, the fluorescent lifetime of $\approx 100 \mathrm{~nm}$ sized FND is $\approx 30-40 \mathrm{~ns}$ (compared to $12.7 \mathrm{~ns}$ in bulk). Such value is one order of magnitude longer than tissue autofluorescence lifetime ( $\approx 1-2 \mathrm{~ns})$. This offers a simple way to enhance the FND signal-to-background ratio by combining pulsed laser excitation with time-gated detection (6). Altogether, FND gather a unique set of properties that makes them particularly suited tools for stem cell and regenerative medicine researches, because these fields require to track cell proliferation and differentiation on long-term scale (8).

Stem cells are non-differentiated cells that can differentiate in specialized cell types and can go through numerous cycles of cell division. For these reasons, therapy based on stem cell injection or transplantation have been developed to treat a wide range of high prevalence diseases, including cancers and neurodegenerative diseases. To optimize stem cell therapy, it is crucial to track stem cells fate in the organism where they have been transplanted. Several strategies are used to label stem cells in vivo (17), including (i) the over-expression of a reporter gene and the subsequent labeling of the corresponding protein with a molecular probe associated to either a radiotracer (then imaged by PET or SPECT), or a fluorescent dye; and (ii) internalization of magnetic or fluorescent nanoparticles into stem cells that allow them to be traced by MRI or photonic imaging. The genetic transformation of cells and their subsequent labeling is a powerful but complex approach with cell viability issues and risks of mutagenesis. Labeling with nanoparticles can offer long signal persistence which represents a significant advantage. In this category, fluorescent NDs have been successfully used to label lung stem cells and track their engraftment and regenerative capability in mice (18). FND-labeled lung stem cells 
(LSCs) were injected intravenously into lung-injured mice, after having checked that FND labeling does not suppress the cells properties of self-renewal and differentiation into pneumocytes. Mice were sacrificed at different time points and organs were collected and sectioned to evaluate the capability of LSC to stimulate lung regeneration by its engraftment and differentiation. Raster scanning timegated fluorescence (TGFluo) confocal microscopy (6) and fluorescence lifetime imaging (FLIM) allowed to unambiguously identify FND-labelled LSCs despite tissue immunostaining and autofluorescence. Figure 1 displays lung section immunochemical staining, FLIM and TGFluo images of a mouse sacrificed 7 days after LSCs injection. Immunostaining revealed that club cells, which are protective secretory cells in the epithelium of terminal and respiratory bronchioles, have regenerated after their initial ablation with naphthalene. Moreover, TGFluo and FLIM showed that LSCs preferentially engraft in injured bronchioles (compared to alveoli and to bronchioles of non-injured mice) where they participate to club cells regeneration (Figure 1b). This proof of principle experiment (18) showed that FND-labeling of stem cells, combined to TGFluo and FLIM, allows in-depth investigation of the optimal conditions of engraftment and regeneration in the targeted tissue. The method was recently extended to monitor the homing of human mesenchymal stem cells (MSCs) in miniature pigs (19), the respiratory system of this animal having a human-like immune responses. MSCs are self-renewing, multipotent progenitor cells with the capacity to differentiate into distinct mesenchymal lineages. In human, MSCs are mainly found in bone marrow, adipose, and placenta tissues. Owing to their multilineage differentiation potential, MSCs are considered as the most promising stem cells for therapy and regenerative medicine, which is a strong motivation to develop reliable methods to measure their biodistribution and pharmacokinetics in vivo in preclinical evaluations. Indeed, such methods will be crucial to determine to which extent the transplanted MSCs home to the target organs, to optimize the treatment and avoid inappropriate differentiation that can lead to cancer in the case of incorrect location. As in the previous study on mice (18), cell viability, immunomodulation and proliferation assays were conducted in vitro, and FNDs uptake by MSCs did not impact any of these properties. Then, for their investigation of the fate of FND-labeled MSCs injected in miniature pigs, Su et al. (19) 
have taken advantage of the unique magnetic field sensitivity of NV-center fluorescence (14), so that under a modulation of an external magnetic field, only the fluorescence intensity of FND varies and not the one of tissue autofluorescence. Therefore, FND signal can be retrieved within a strong autofluorescent background by simple demodulation (15). Using this magnetically modulated fluorescence (MMF) background-free technique, in combination with time-gated fluorescence imaging, Su et al. (19) were able to quantify precisely the amount of FND-containing human placenta choriodecidual membrane-derived MSCs (pcMSCs) in each of the five main organs of miniature pigs. The animals were sacrificed at specific time points after their intravenous injection of FND-labeled pcMSCs. Their main organs were then extracted, digested with acids under high pressure, and FND fluorescence was precisely quantified in each of them, thanks to MMF technique. Up to $70 \%$ of the FND-labeled pcMSCs were found to reside in the lungs at 24-48 hours after FND-labeled pcMSCs injection. In contrast, using free FND lead to only $25 \%$ of them ending in the lungs. The next most important fraction was found in the liver ( $\approx 2 \%$ in both FND-labeled MSCs or free FND). This quantitative information could only be obtained, for the first time, thanks to the unique magneto-optical properties of NV centers in FNDs. The combination of MMF and FLIM/TGFluo $(18,19)$ represents a promising alternative to radioisotope labeling commonly used in stem cell tracking applications, at the single cell levels (17). Noteworthy, FND does not alter the differentiation of both mouse and human embryonal carcinoma stem cells into neurons in vitro, as induced by conventional protocols (20).

The perfect photostability, absence of toxicity, and high fluorescence brightness of FNDs are also key properties to track rare cells such as circulating tumor cells or cancer stem cells (CSCs). The later belong to a sub-population of tumor cells that are resistant to current chemotherapy and radiotherapy treatments. Despite growing evidence of CSCs implication in tumor growth and recurrence, their isolation and eradication are still a challenge (21). Conventional fluorescent markers are not chemically and photophysically stable enough to track CSCs on the few-days (up to one month) period required for diagnosis and prognosis applications. Lin et al (22) have shown that FND are promising labels for such a goal. They have used a human breast cancer cell line established from a patient, and selected 
for their great capacity to form mammospheres (clumps of mammary gland cells), an indication for the presence of a stem cell population. Lin et al (22) have discovered in in vitro assays that there are two populations of cells having spontaneously uptaken FNDs, a population $\mathrm{FND}^{+}$with high content of FNDs, and another one FND- with a low content. Incidentally, the fluorescence signal from FND- cells, as measured by flow cytometry, decreased faster than the one of $\mathrm{FND}^{+}$. This indicates that $\mathrm{FND}^{+}$may belong to a slowly proliferating, quiescent cell subpopulation, possibly corresponding to a CSC phenotype. FNDs labeling was also compared to current CSC fluorescence labeling methods, and was found to outperform them in terms of (i) absence of genotoxicity and (ii) longer term tracking capability owing to a longer retention in cells (22). This proof of principle experiment demonstrated the potential use of FND to track and find quiescent human CSCs.

We have shown that cell labeled with cytoplasmic FNDs can be retrieved in an organism on a longterm scale, even at the single cell level and in a highly autofluorescent environment. While such experiments are carried out on sacrificed animals, detection of such FND-labeled cells in a living organism is more challenging. However, Hui et al (23) have extended the raster scanning time-gated fluorescence detection of FND to dynamical wide-field imaging. To this aim, they used an intensified CCD array detector and triggered its gate opening with some delay relative to the excitation lase pulse. This setup allowed them to track FND-labeled mouse lung cancer cell motion at video rate in the blood stream of a living mouse after injection in its vein (Figure 2). Fluorescence imaging was then carried out near one of the main blood vessels of the mouse ear. The autofluorescence (from sebaceous glands) was still present in TGFluo images, but it can be readily removed by post-processed background subtraction, so that the motion of FND-labeled cells can be detected with a large signal-to-background ratio, which facilitates the trajectory extraction (Figure $\mathbf{2 c}$ ). One potential application of TGFluo widefield microscopy of FND could be single particle tracking in a complex environment such as brain . In section 3 of this chapter, we present an alternative carbon-based nanomaterial, i.e. single-wall carbon nanotube, that is tracked individually thanks to its emission in the near-infrared tissue transparency window, in order to probe the extracellular nanoscale environment in an intact brain tissue. 
In this section, we have shown that FNDs, thanks to (i) their remarkable photophysical and magneto-optical properties that allow background-free imaging, and to (ii) their very low cellular toxicity, are very promising labels to optimize stem cell therapy protocols in preclinical research. Several studies have shown that FNDs can provide quantitative data that are hardly accessible by other methods. Furthermore, FND-labeled cells can also be tracked individually in real time with backgroundfree wide-field video-microscopy, offering appealing prospects to identify rare cells like circulating tumor cells, directly in blood circulation.

\section{Monitoring inter and intra cellular dynamics with fluorescent nanodiamonds}

In the previous section, we have discussed several studies that filled up cells with FNDs and track their fate in organism like mouse or pigs. However, FND can also be tracked at the single particle level in semi-transparent organisms such as zebrafish (24), C-elegans (25) or drosophila embryos (26). The main motivation was that bright near-infrared emitting FND reporters might help to understand the inner dynamics of a developing organism. For example, in Chang et al (24), $100 \mathrm{~nm}$-size FNDs, coated with bovine serum albumin (BSA) to prevent aggregation, were microinjected into the yolk of a zebrafish embryo at the one-cell stage. They found that the FNDs underwent unidirectional and stopand-go traffic in the yolk cell with an average velocity of $0.3 \mu \mathrm{m} / \mathrm{s}$. Moreover, when incorporated into dividing cells, these particles could migrate into the fish's body as the embryos developed from larvae into adult fish. Interestingly, fishes injected with FND did not present abnormalities at the adult stage, meaning that FNDs did not interfere with embryogenesis.

The first attempt to track single FNDs in a whole organism was made by Igarashi et al. (27). They developed a selective imaging method based on ODMR (cf section 1 for definition) to improve the image contrast of FNDs in vivo. Briefly, two wide-field fluorescence images were recorded with or without a microwave (MW) modulation at the ODMR resonant frequency of $2.87 \mathrm{GHz}$. Subtracting two images (with MW on and MW off) pixel-by-pixel allowed them to remove the autofluorescence which is insensitive to $\mathrm{MW}$, and provide images disoplaying of FNDs alone. Then, they performed long-term tracking of single FNDs in both C. elegans and mice. In a following paper, they improved even further 
the temporal resolution of their apparatus (sampling rate increased up to $20 \mathrm{kHz}$ ) by using a spectrometer equipped with an avalanche photodiode (28). As a proof-of-principle, they simply injected FNDs into the intestine of $C$. elegans and performed real-time recording of FND without background fluorescence. Although the experiments reported in these articles were not addressing specific biological challenges, this selective imaging technique could be implemented for other living organisms and bring unexpected findings.

As previously discussed in section 1, time-gated imaging is an alternative approach to achieve background-free detection of FNDs in whole organisms. For example, Kuo et al. (25) used it to investigate the intercellular transport of yolk lipoproteins in $C$. elegans (

(a1)

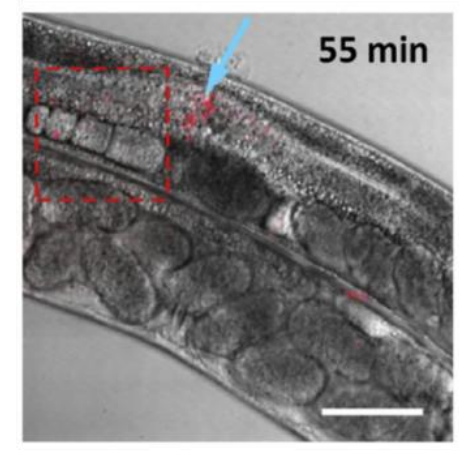

(b1)

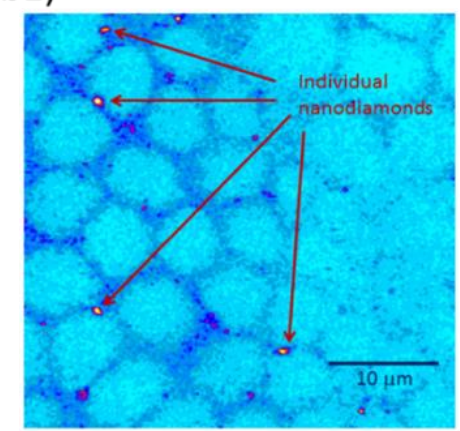

(a2)
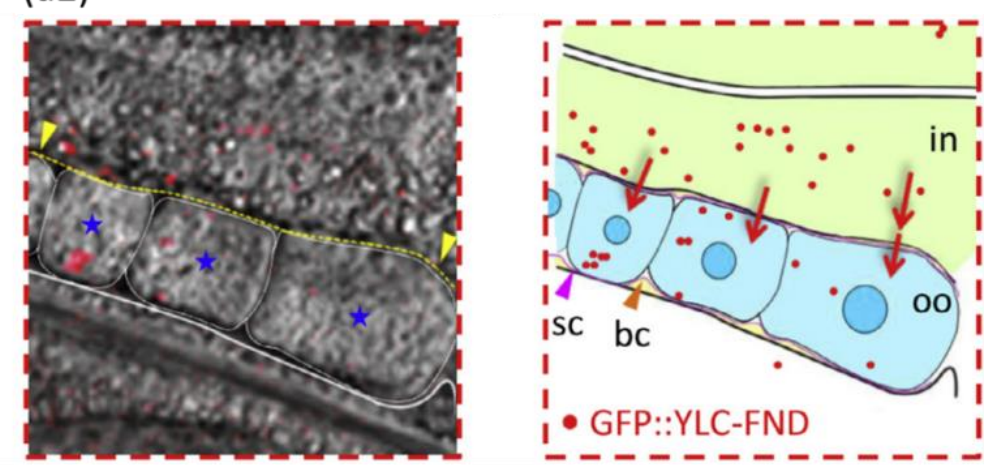

(b2)

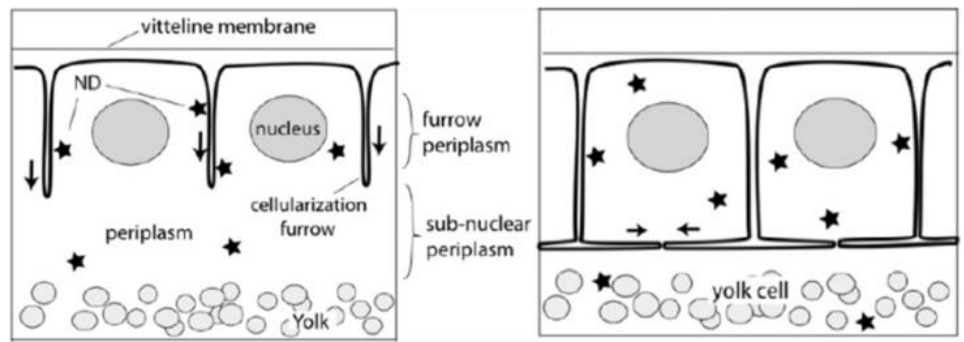

Figure 3a). FNDs were first non-covalently coated with green fluorescent protein (GFP)-tagged yolk lipoprotein complexes (YLC) to for the GFP::YLC-FNDs platform. Functionalized FND were then microinjected into the anterior intestinal cells near the pharynx. Worms were examined individually 
to identify the location of GFP::YLC-FNDs in the specific cells or organs over $55 \mathrm{~min}$ after injection (

(a1)

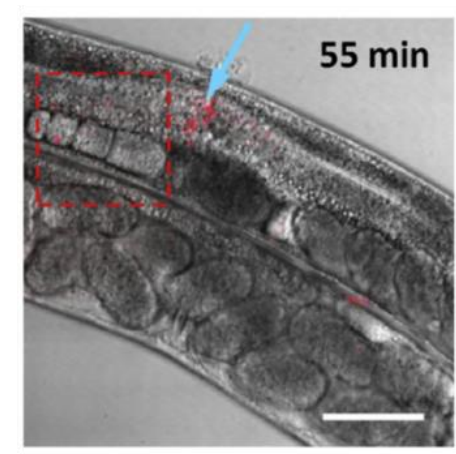

(b1)

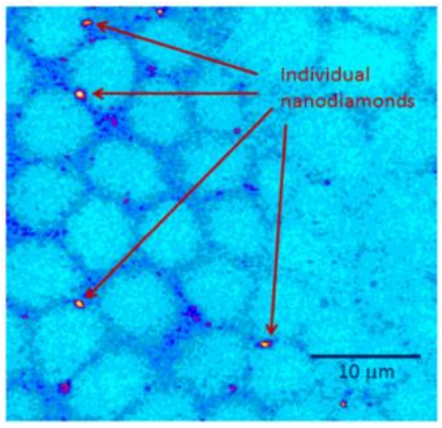

(a2)
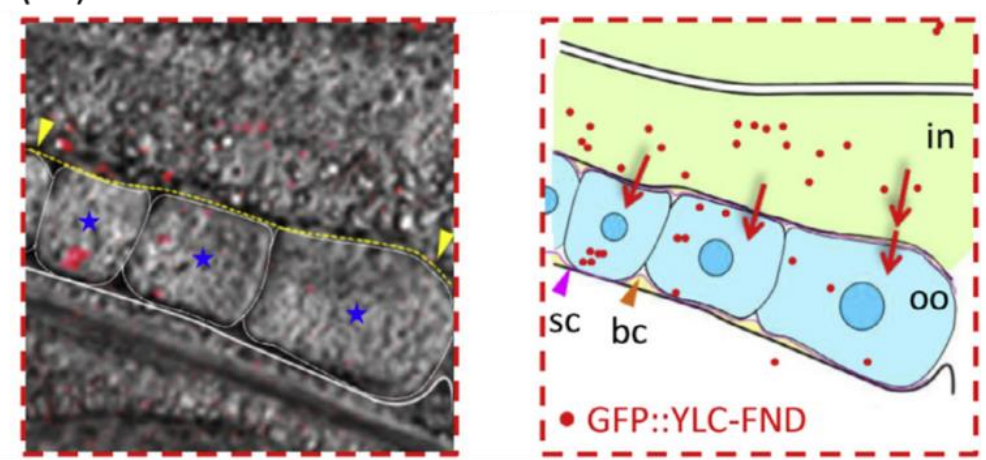

(b2)

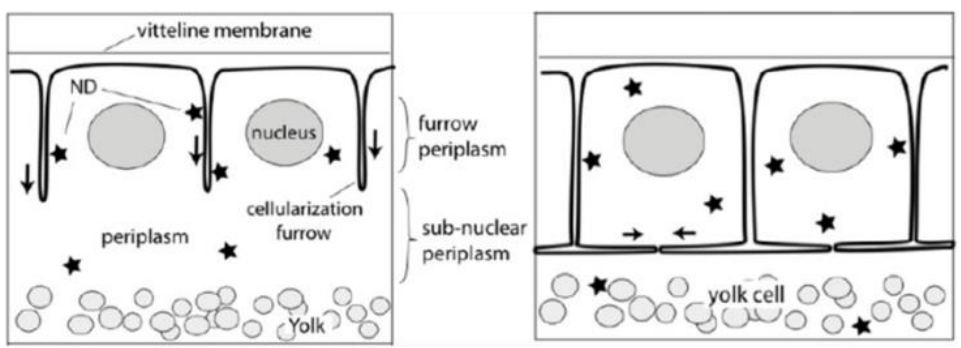

Figure 3a). Results showed that the functionalized FNDs appeared in the posterior intestine immediately after administration. Then, they migrated into the pseudo-coelom and the loop region of the gonad at $5 \mathrm{~min}$ and $12 \mathrm{~min}$ post-injection, respectively. At 20 min post injection, they became visible in the oocytes. Interestingly, a FND-containing embryo was found at 55 min post-injection, indicating that oocytes can be fertilized and can normally develop into embryos.

Another example of SPT of FNDs inside living organisms is the study of the embryogenesis in the drosophila (26). In this article, the author introduced BSA-coated FNDs into the embryo and 
investigated the FND diffusion in both furrow periplasm and sub-nuclear periplasms by SPT

(a1)

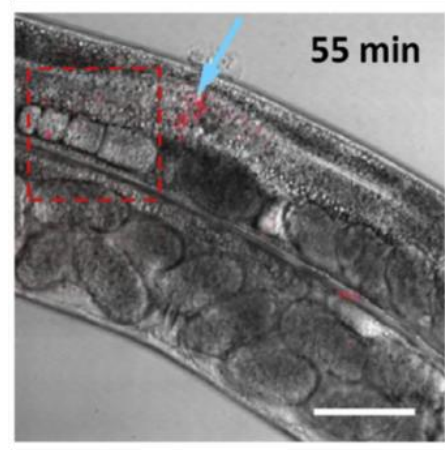

(b1)

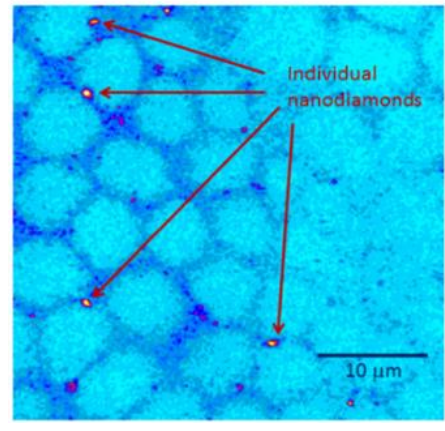

(a2)
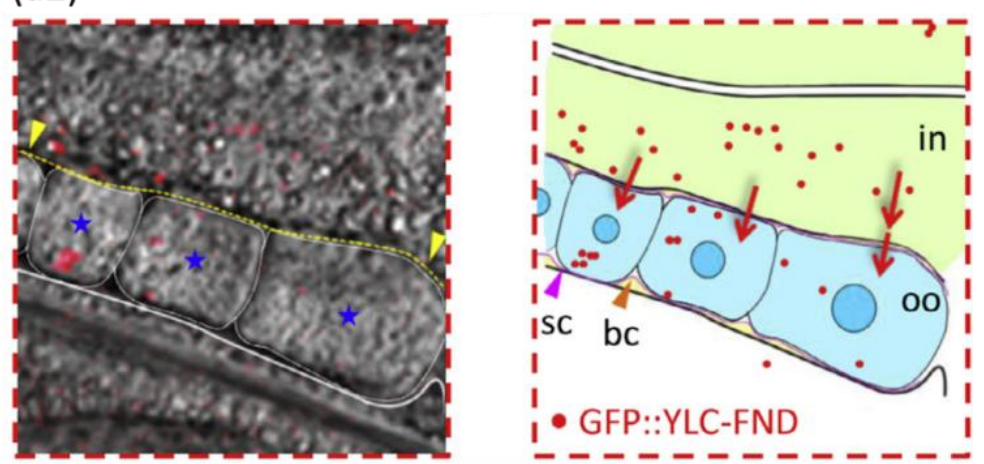

(b2)

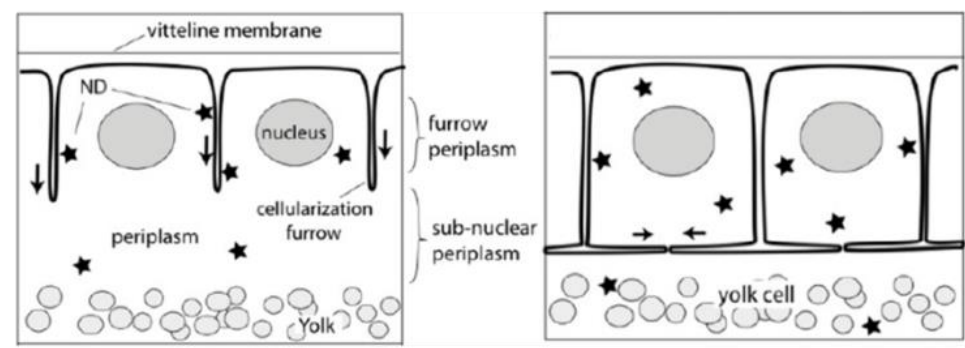

Figure 3b). They observed that during cellularization at the posterior end of the drosophila embryos, the internalized FNDs in the blastoderm cells displayed two regimes of motion: free diffusion and molecular motor-driven motion. By tracking the FNDs and extracting the trajectories, the authors determined a mean diffusion coefficient of $0.006 \mu \mathrm{m}^{2} / \mathrm{s}$ in the furrow and $0.06 \mu \mathrm{m}^{2} / \mathrm{s}$ in the subnuclear periplasm. Although the velocity in these two compartments were similar $(0.13 \mu \mathrm{m} / \mathrm{s}$ and $0.27 \mu \mathrm{m} / \mathrm{s}$ respectively), the mean diffusion coefficient differs by one order of magnitude. The author therefore concluded that cytoskeletal networks in the furrow periplasm in more compact that in the sub-nuclear periplasm.

Although we previously described the use of FND tracking in fixed (18) or living organisms (25-27), the seminal article that introduced FNDs in biology a decade ago actually showed that these fluorescent particles can be used to study intracellular dynamics $(29,30)$. This approach requires to internalize single FNDs inside a cell and track them over time using a diffraction-limited optical system. The first proof of principle was done in cancer cell line, which are easy to grow and maintain. Moreover, due to their intrinsic proliferative behavior, exogeneous compounds are easy to internalize by 
endocytosis. In a subsequent article, the same group showed that bright $35 \mathrm{~nm}$-sized FNDs can be tracked in 3D in a reproducible manner (12). Figure 4a displays a typical result of a bare FND moving inside a HeLa cell.

Another application of SPT of FNDs inside living cells is described in Liu et al (31). In this work, FNDs were conjugated to the transforming growth factor (TGF) in order to target the TGF- $\beta$ membrane receptors and track their motion in 3D. The authors showed that the TGF- $\beta$ receptor presents three different regimes of motion: immobile, intermediate, and fast diffusion (Figure $4 b$ ). After treatment with small molecule kinase inhibitors (SMI), the fraction of the immobile population significantly decreased compared to the one of intermediate and fast populations. This result is consistent with the fact that SMI releases the TGF- $\beta$ receptor from a larger binding complex, therefore increasing its diffusion on the membrane. The author claimed this data proves that immobilized TGF- $\beta$ is essential for active signaling. Overall, this work shows that FNDs can be used as specific tagging nanotool of endogenous proteins in living cells for the study of transmembrane signaling dynamics.

Interestingly, by recording the ODMR spectra over time, McGuinness et al. (32) were able to track the orientation of a single FND entrapped in a living cell. The author applied a uniform magnetic field to live HeLa cells containing 50-nm FNDs, each of which hosts a single $\mathrm{NV}^{-}$center. By closely analyzing the resonance frequency shift in the ODMR spectra, information on the rotational motions of the FNDs in the cell was inferred. Thanks to the perfect photostability of FNDs, the authors were able to continuously track the orientation change over $16 \mathrm{~h}$.

Until recently, single particle tracking of FND has only been conducted in cancer cell lines. Although, this biological system can be useful for drug discovery or fundamental studies on intracellular dynamics, it falls short to address some other questions like brain disease-related ones requiring culture of neurons dissociated from mouse embryos. A recent study proved that SPT of FND can be easily conducted in neuron culture with a high enough sensitivity to detect intraneuronal transport abnormalities (1). Previous attempts to quantify intraneuronal transport have been using fluorescent proteins to label organelles (33). However, this approach faces several limitations: low 
transfection yield, uncontrolled protein expression, photobleaching, and cytotoxicity. Fluorescent semiconductor nanocrystals (quantum dots, QDs) is another type of fluorescent reporter that was used to monitor various cellular events (34), but attempts to use QDs to study intraneuronal transport have been limited to the specific model of long axons of dorsal root neurons cultured in microfluidic devices (35). Moreover, QDs blinking impedes high spatiotemporal resolution tracking and therefore biases transport parameter measurements (1). Considering the intrinsic limitations of other potentially useful reporters, FND unique properties of high brightness, photostability and absence of cytotoxicity, make them a tool of choice to detect abnormalities of intraneuronal transport.

Haziza et al (1) has developed a novel quantitative assay based on tracking of $30 \mathrm{~nm}$-sized FNDs in mouse hippocampal neurons (Figure 5a). They took advantage of the endocytosis mechanism to introduce FNDs into neuronal branches and recorded FND trajectories with Total Internal Reflection Fluorescence (TIRF) video microscopy at $50 \mathrm{~ms}$ temporal resolution, giving a spatial localization accuracy of $30 \mathrm{~nm}$. They were then able to reconstruct the entire trajectories with a tracking software (Figure 5b). Using a custom-made algorithm, they extracted relevant transport readouts: velocity (in $\mu \mathrm{m} / \mathrm{s}$ ), run length (in $\mu \mathrm{m}$ ), processivity (in seconds), pausing time (in seconds), pausing frequency (events per minute) and the diffusion coefficient (in $\mu \mathrm{m}^{2} / \mathrm{s}$ ). Interestingly, they proved the superiority of FND over QD to reliably report transport parameters and therefore emphasizes that the perfect photostability of FND is key for this approach (1). To prove the sensitivity of the FND-tracking assay, the author designed three experiments with increasing complexity. They first used a pharmacological drug that impact the microtubule-based intraneuronal transport, but at nanomolar concentrations. Then, as a validation of the ability of their technique to record abnormal intraneuronal transport, they incubated neuron culture with sub-micromolar concentrations of amyloid- $\beta(A \beta)$ peptide, a wellknown molecular player Alzheimer's in disease $(36,37)$ (Figure 5c). This experiment suggests that the FND-tracking assay could be used to screen drugs capable of rescuing Alzheimer's disease phenotype. Eventually, as a proof-of-principle, they applied their method on two transgenic mouse lines that mimic small variation $(\approx+30 \%)$ in protein concentration found in brains of patients $(1)$. In both cases, 
the FND-tracking assay was sensitive enough to detect small modifications of the intraneuronal transport parameters in the transgenic neurons.

In this section, we have discussed the use of FND at the single particle level. Single FND tracking can be used in the whole organism like C-elegans or drosophila embryos and led to important findings on the developmental organization. In addition, SPT of FND can be performed at the single cell levels. Historically done in cancer cell lines for basic research, the unique photophysical properties of FND and their biocompatibility brought them into the field of neurosciences and brain diseases. A novel FND-tracking assay that monitor the endosomal transport inside neuronal branches was recently reported. It was proved to be sensitive enough to detect subtle change in gene expression, as the ones found in the brain of patient with autism or Alzheimer's disease. For the first time, a nanoparticlebased assay is able to directly measure a functional impact of genetic risk factors found in patient with brain disease, opening the door for applications in translational nanomedicine.

\section{Single wall carbon nanotube: a near-infrared optical label to probe nanoscale extracellular} environments in live brain tissue.

Some of the current challenges for a better understanding of fundamental processes in biology and for applications in medical science concern the development of imaging nanoprobes and chemical nanosensors which are not limited to single cell studies, but applicable within live intact tissues. Nanoprobes and nanosensors must ideally display intense and specific signals reporting (related to) local physical and/or chemical environments. The elaboration of new drug delivery strategies shares similar requirements since drug transporters must be able to carry multiple functions inside living organisms including (i) the recognition of a specific cellular/molecular target, (ii) the actuation of the drug itself and if needed, (iii) the signalization of their tissue localization. Whether we are dealing with drug transporters, imaging nanoprobes or chemical nanosensors, all of them thus need to display excellent accessibility within intact tissues.

For these applications, the development of spherical particles as multifunctional objects encounters contradictory requirements: they should be small enough for tissue accessibility and large 
enough for carrying the multiple functions mentioned above, i.e. targeting capabilities and/or drug delivery, chemical sensing signals and imaging contrast through tissues . Therefore, the combination of optical, chemical and penetration capabilities in tissue are usually not fulfilled together with spherical nanoparticles. Because they display extreme length-to-diameter aspect ratios as well as unique optical/chemical/mechanical properties, single walled carbon nanotubes (SWCNTs) constitute a promising platform to fulfill these requirements.

SWCNTs distinguish themselves from double-walled carbon nanotubes and multi-walled carbon nanotubes by their single-layer cylindrical sidewall structure, which provides them more finely tuned physical and chemical properties for applications. They are characterized by the thinnest diameters $(\approx 1-3 \mathrm{~nm})$ and have lengths of hundreds of nanometers up to few micrometers, conferring them unusual aspect ratios. SWCNTs display strong optical resonances and the discovery of their nearinfrared (NIR) luminescence $(38,39)$ from semiconducting species paved the way to their detection at the single nanotube level in liquid environments (40-42). Individual semiconducting SWCNTs exhibit luminescence with large Stokes shifts in the NIR window (typically ca. 900 to $1400 \mathrm{~nm}$ ) which falls in the therapeutic window of biological tissues (typically. 650 to $1400 \mathrm{~nm}$ ), and is thus potentially free of autofluorescence coming from biological structures. SWCNT optical properties, which are based on excitonic processes are now rather well understood (43-45). Despite their low luminescence quantum efficiencies (46-48), water solubilized SWCNTs display exceptionally high luminescence rates for a single nano-object (41) due to remarkably large absorption cross-sections (49-53). They also display exceptional luminescence signal stabilities (tens of $\min (54)$ ) with negligible blinking and photobleaching. Using suitable encapsulation strategies, fluorescence emission of SWCNTs can be highly stable, allowing long-term imaging in aqueous environments at the single nanotube level $(42,54)$, but also intracellular tracking of single nanotube in cultured cells (55-57). Interestingly, depending on their encapsulating agents, SWCNT photoluminescence can be extremely sensitive to their chemical environment $(42,58,59)$ which opened the way to sensing capabilities in biological environments (60-64). Moreover, their brightness, photostability and spectral imaging range $(38,65-$ 
67) make SWCNTs unique probes at the ensemble level for imaging the whole animal vascular systems $(68,69)$.

In the following, we will describe how the unique combination of SWCNT diffusion and optical properties within complex environments can be exploited at the single nanotube level in order to access live intact tissue structures and probe their nanoscale environment. Diffusion and optical imaging being inherent to the aforementioned applications, it will be possible to extend the following concepts to other developments such as chemical nanosensors, optical contrast agents or drug delivery systems within living biological tissues.

Molecular diffusion processes are key for the function of living matter $(70,71)$. However, they occur within complex environments characterized by heterogeneous nanoscale organizations and chemical contents, which make it difficult to dissect. In this context, single molecule optical microscopy has been crucial over the last decades to study the spatio-temporal heterogeneity of the biological complexity. It provides the ability to probe the full populations of diffusive behaviors including the presence of minority populations (e.g. localized, anomalous or Brownian diffusion), in multiple environments spanning from nanometer- to micrometer-scale resolution. A widely used approach to study molecular diffusion is the super-localization technique, based on the determination of a diffraction-limited emitter position with nanometer precision $(72,73)$ Because biological constituents are generally neither detectable nor resolvable at the molecular scales, single molecule microscopy commonly uses highly luminescent nano-emitters which probe their biological structures while being efficiently detected by an optical microscope. This is usually performed in isolated cells or very thin tissue preparations (up to a few micrometers) at visible or eventually far-red wavelengths using organic dyes, autofluorescent proteins or eventually small emitting nanoparticles. For application in thick intact biological tissues, scattering and absorption of visible light complicates single molecule detection such that single-molecule imaging within samples thicker than isolated cells is only emerging and mostly limited to a few cellular layers $(74,75)$. To access the optical window of biological tissues, i.e. where 
scattering and absorption of tissue are minimal, the use of nano-probes with optical response in the NIR is thus crucial and SWCNTs match this requirement.

Prior to their use as biological nanoprobes, SWCNTs have to be biocompatible. In liquid environment, the photoluminescence of SWCNT is obtained by coating their surface with surfactants or polymers. Although surfactants like sodium dodecylbenzene sulfonate or bile salts are known to provide the best luminescing SWCNTs in aqueous environments $(76,77)$, their use for biological applications should be avoided as they alter the integrity of cellular membranes. Several biocompatible coatings were investigated and among them, phospholipid-polyethylene glycol (PL-PEG) provides high luminescence, low toxicity (68) as well as minimal non-specific interaction with cellular membrane (78). The use of PL-PEG allows long term imaging directly within live tissue (2) and long circulation time in the vascular system in vivo (69). For application where interactions with cellular constituent are sought, in particular for sensing applications, the use of short DNA sequences $(60,62)$, biomolecules $(61)$ or biocompatible polymers $(63,64,79)$ have provided interesting biocompatible routes for suspending luminescent SWCNT with sensing capabilities.

Several wavelengths that fall in the biological "transparency" window can be used to excite SWCNTs in tissues. Considering the widely used $(6,5)$ SWCNT chirality ${ }^{1}$, Danné et al $(80)$ investigated the possibility to excite them in brain tissues at the single nanotube level by continuous-wave (cw) lasers at their second-order excitonic transition $(568 \mathrm{~nm})$, their K-momentum exciton-phonon sideband $(845 \mathrm{~nm})$ or through up-conversion $(1064 \mathrm{~nm})$. The effects of tissue scattering, absorption, autofluorescence and temperature increase induced by excitation light were systematically examined. Video rate imaging of single $(6,5)$ SWCNTs diffusing in liquid environments upon cw up-conversion excitation at $1064 \mathrm{~nm}$ was first demonstrated and the comparison between different excitation

\footnotetext{
${ }^{1}$ The chiral indices $(n, m)$ of a SWCNT symbolize the nanotube structure, i.e. how a graphene sheet is rolled-up along the "chiral vector" in the basis of the graphene sheet to form the nanotube. Each SWCNT chirality displays specific electronic and optical properties.
} 
wavelength to obtain identical photoluminescence rates from single biocompatible PL-PEG coated $(6,5)$ SWCNTs was performed. This allowed a realistic determination of the best excitation strategy to detect single $(6,5)$ SWCNTs in live brain tissue. Danné et al $(80)$ found that despite its most red-shifted wavelength, care should be taken when up-conversion aims at being used to image single SWCNT at video-rate in live tissues because of the temperature rise induced by laser absorption of the tissue. They concluded that excitation at the K-momentum exciton-phonon sideband ( $845 \mathrm{~nm}$ ) is currently the optimal strategy for imaging single $(6,5)$ SWCNTs while further fundamental understanding of the processes underlying up-conversion of SWCNTs might help optimizing excitation efficiencies of single SWCNTS.

To serve as biological nano-probes in tissues, SWCNT long 1D shape inevitably raised concerns about tissue penetration. A first insight about their potential for tissue penetration was provided through the discovery that long SWCNTs can move by reptation or "snake-like motion" in crowded environments (54) (Figure 6). The extent of SWCNTs diffusion in such environments is indeed function of their length, diameter and of environment crowdedness (2). More precisely, the persistence length $\left(L_{p}\right)$ or rigidity of SWCNTs, depends on their diameter and was previously characterized using thermal bending statistics (81). It ranges from a few tens of $\mu \mathrm{m}$ up to several hundreds of $\mu \mathrm{m}$. It follows that when SWCNTs are immersed in crowded environments, they undergo Brownian or random thermal motion according to two distinct diffusive regimes depending on their length, persistence length and the network characteristic dimension (६) (54). A first regime, proposed by Odijk (82) takes place when the SWCNT length is larger than the independent deflection length $\lambda=\left(L_{p} \xi^{2}\right)^{1 / 3}$, in which case SWCNTs will undergo reptation as introduced by de Gennes (83). A second regime, predicted by Doi (84), happens when SWCNTs length is smaller than the independent deflection length $\lambda$. For Doi regime, in contrast to Odijk regime, the diffusion is completely independent of the flexibility and can be modeled assuming fully rigid SWCNTs. The transition between these two regimes were observed experimentally by Fakhri et al (54) by analyzing the diffusion of individual SWCNTs of different rigidities (i.e. different diameters) and lengths in aqueous gels. This types of behaviors later lead to the observation obtained 
at the ensemble level by Jena et al (85), that SWCNT unusual aspect ratios contribute to enhance their penetration within multicellular tumor spheroids, but this enhancement was also found dependent on the encapsulation strategy $(85,86)$. Godin et al (2) pushed these concepts a step further by performing single nanotubes studies in live brain tissues. They showed that the analysis of the movement of single PL-PEG coated SWCNTs can be used to unravel the extracellular dimensions and fluidity of live brain tissues. They indeed demonstrated that upon injection into the lateral cerebroventricles of young rat brains, PL-PEG coated SWCNTs diameters were small enough to enabling diffusion into small brain compartments, but at the same time, that the SWCNT length is sufficiently long to slow down SWCNT movements and allow them to extensively explore their submicroscopic environment. In addition, due of their photostability and NIR emission properties, single SWCNTs could be imaged in the extracellular space of acute brain slices in different areas of the sagittal brain, at imaging depths of a few tens of $\mu \mathrm{m}$ up to $\sim 100 \mu \mathrm{m}$. Their movements could be tracked for long periods of time at video rate, allowing recording trajectories that are orders of magnitude longer than trajectories obtained by tracking small isotropic probes (e.g. dye molecules, antibodies, latex beads and quantum dots). Super localization of the nanotube positions provided the first super-resolved maps of the extracellular space of live brain tissues (down to $40 \mathrm{~nm}$ resolution, Figure 7), demonstrating that the extracellular space is larger than previously thought in live brain, as recently suggested by electron microscopy in cryo-fixed tissues (87). In addition, single SWCNT tracking in live tissues revealed the heterogeneity of the extracellular space local viscosity and its dependence on extracellular matrix components.

Altogether, these findings indicate that SWCNT constitutes a new platform to envision a better understanding of the extracellular space of live tissues, through the combination of unique tissue accessibility, optical properties for deep tissue nano-imaging, but surely in future for local chemical sensing capabilities in local tissue areas. These new nano-reporters based on SWCNTs will confidently disseminate in biology, grounding new fundamental concepts in organ development, physiology and pathophysiology. We can also envision that such concepts can be applied for the development of novel drug delivery strategies based on 1D nanoscale transporters. 


\section{Conclusion}

In this chapter, we have highlighted recent works that applied photoluminescent carbon nanomaterials, mainly fluorescent nanodiamonds (FND) and single-wall carbon nanotubes (SWCNT), to dissect cellular and organism dynamics. The unique combination of SWCNT diffusion and optical properties can be exploited at the single nanotube level to probe nanoscale environment in living tissues, such as mouse brain. Although one can argue that FNDs might suffer from their too large size (typically $30-100 \mathrm{~nm}$ ), several groups have made progress to reduce it down to $10 \mathrm{~nm}$ without significantly compromising their photophysical properties $(88,89)$. In fact, researchers are benefiting from the perfect photostability, the unique magneto-optical property, the easy surface chemistry activation, and the absence of toxicity of FNDs and thoroughly used them at different biological scales. FNDs can help tracking cells in organisms like mouse or miniature pigs $(18,90)$, or used to monitor membrane receptor dynamics (31) and intraneuronal transport (1). They may also serve as gene and drug delivery vehicles by modifying their surface chemistry $(91,92)$. Altogether, despite many challenges, carbon nanomaterials have proven themselves as highly promising imaging tools to screen cellular and organism dynamics. The next step would be to translate these methodologies and findings from labs to clinical applications such as diagnosis and therapy.

\section{REFERENCES}

1. Haziza S, Mohan N, Loe-Mie Y, Lepagnol-Bestel A-M, Massou S, Adam M-P, et al. Fluorescent nanodiamond tracking reveals intraneuronal transport abnormalities induced by braindisease-related genetic risk factors. Nat Nanotechnol. 2017;12(4):322-8.

2. Godin AG, Varela JA, Gao Z, Danné N, Dupuis JP, Lounis B, et al. Single-nanotube tracking reveals the nanoscale organization of the extracellular space in the live brain. Nat Nanotechnol. 2016 Nov 21;12(3):238-43.

3. Tan L, Wu T, Tang Z-W, Xiao J-Y, Zhuo R-X, Shi B, et al. Water-soluble photoluminescent fullerene capped mesoporous silica for $\mathrm{pH}$-responsive drug delivery and bioimaging. Nanotechnology. 2016 Aug 5;27(31):315104.

4. Zhang $\mathrm{H}$, Zhang $\mathrm{H}$, Aldalbahi A, Zuo X, Fan C, Mi X. Fluorescent biosensors enabled by graphene and graphene oxide. Biosens Bioelectron. 2017 Mar;89:96-106.

5. Wang J, Qiu J. A review of carbon dots in biological applications. J Mater Sci. 2016 May 10;51(10):4728-38.

6. Faklaris O, Garrot D, Joshi V, Druon F, Boudou J, Sauvage T, et al. Detection of Single 
Photoluminescent Diamond Nanoparticles in Cells and Study of the Internalization Pathway. Small. 2008 Dec;4(12):2236-9.

7. Faklaris $O$, Joshi V, Irinopoulou T, Tauc $P$, Sennour M, Girard H, et al. Photoluminescent Diamond Nanoparticles for Cell Labeling: Study of the Uptake Mechanism in Mammalian Cells. ACS Nano. 2009 Dec 22;3(12):3955-62.

8. Fang C-Y, Vaijayanthimala V, Cheng C-A, Yeh S-H, Chang C-F, Li C-L, et al. The Exocytosis of Fluorescent Nanodiamond and Its Use as a Long-Term Cell Tracker. Small. 2011 Dec 2;7(23):3363-70.

9. Huang Y-A, Kao C-W, Liu K-K, Huang H-S, Chiang M-H, Soo C-R, et al. The effect of fluorescent nanodiamonds on neuronal survival and morphogenesis. Sci Rep. 2014 Nov 5;4:6919.

10. Simpson DA, Morrisroe E, McCoey JM, Lombard AH, Mendis DC, Treussart F, et al. NonNeurotoxic Nanodiamond Probes for Intraneuronal Temperature Mapping. ACS Nano. 2017 Dec 26;11(12):12077-86.

11. Paget V, Sergent J a, Grall R, Altmeyer-Morel S, Girard HA, Petit T, et al. Carboxylated nanodiamonds are neither cytotoxic nor genotoxic on liver, kidney, intestine and lung human cell lines. Nanotoxicology. 2013 Nov 22;5390:1-11.

12. Chang Y-R, Lee H-Y, Chen K, Chang C-C, Tsai D-S, Fu C-C, et al. Mass production and dynamic imaging of fluorescent nanodiamonds. Nat Nanotechnol. 2008 May 27;3(5):284-8.

13. Dantelle G, Slablab A, Rondin L, Lainé F, Carrel F, Bergonzo P, et al. Efficient production of NV colour centres in nanodiamonds using high-energy electron irradiation. J Lumin. 2010 Sep;130(9):1655-8.

14. Doherty MW, Manson NB, Delaney P, Jelezko F, Wrachtrup J, Hollenberg LCL. The nitrogenvacancy colour centre in diamond. Phys Rep. $2013 \mathrm{Jul} ; 528(1): 1-45$.

15. Sarkar SK, Bumb A, Wu X, Sochacki K a, Kellman P, Brechbiel MW, et al. Wide-field in vivo background free imaging by selective magnetic modulation of nanodiamond fluorescence. Biomed Opt Express. 2014 Apr 1;5(4):1190-202.

16. Greffet J-J, Hugonin J-P, Besbes M, Lai ND, Treussart F, Roch J-F. Diamond particles as nanoantennas for nitrogen-vacancy color centers. arXiv.org. $2011 \mathrm{Jul}$ 3; Quantum ph(18):1-4.

17. Nguyen PK, Riegler J, Wu JC. Stem Cell Imaging: From Bench to Bedside. Cell Stem Cell. 2014 Apr;14(4):431-44.

18. Wu T, Tzeng Y-K, Chang W-W, Cheng C-A, Kuo Y, Chien C, et al. Tracking the engraftment and regenerative capabilities of transplanted lung stem cells using fluorescent nanodiamonds. Nat Nanotechnol. 2013 Sep 4;8(9):682-9.

19. Su L-J, Wu M-S, Hui YY, Chang B-M, Pan L, Hsu P-C, et al. Fluorescent nanodiamonds enable quantitative tracking of human mesenchymal stem cells in miniature pigs. Sci Rep. $2017 \mathrm{Mar}$ 30;7(February):45607.

20. Hsu T-C, Liu K-K, Chang H-C, Hwang E, Chao J-I. Labeling of neuronal differentiation and neuron cells with biocompatible fluorescent nanodiamonds. Sci Rep. 2015 May 16;4(1):5004.

21. Batlle E, Clevers H. Cancer stem cells revisited. Nat Med. 2017 Oct 6;23(10):1124-34.

22. Lin H-H, Lee H-W, Lin R-J, Huang C-W, Liao Y-C, Chen Y-T, et al. Tracking and Finding SlowProliferating/Quiescent Cancer Stem Cells with Fluorescent Nanodiamonds. Small. 2015 Sep;11(34):4394-402.

23. Hui YY, Su L-J, Chen OY, Chen Y-T, Liu T-M, Chang H-C. Wide-field imaging and flow cytometric analysis of cancer cells in blood by fluorescent nanodiamond labeling and time gating. Sci Rep. 2014 May 4;4(1):5574.

24. Chang C-C, Zhang B, Li C-Y, Hsieh C-C, Duclos G, Treussart F, et al. Exploring cytoplasmic dynamics in zebrafish yolk cells by single particle tracking of fluorescent nanodiamonds. Adv Photonics Quantum Comput Mem Commun V. 2012;8272:SPIE-SPIE.

25. Kuo Y, Hsu T-Y, Wu Y-C, Chang H-C. Fluorescent nanodiamond as a probe for the intercellular transport of proteins in vivo. Biomaterials. $2013 \mathrm{Nov;34(33):8352-60.}$

26. Simpson D a, Thompson AJ, Kowarsky M, Zeeshan NF, Barson MSJ, Hall LT, et al. In vivo 
imaging and tracking of individual nanodiamonds in drosophila melanogaster embryos. Biomed Opt Express. 2014 Apr 1;5(4):1250.

27. Igarashi R, Yoshinari $Y$, Yokota $H$, Sugi T, Sugihara F, Ikeda K, et al. Real-time background-free selective imaging of fluorescent nanodiamonds in vivo. Nano Lett. 2012 Nov 14;12(11):572632.

28. Yoshinari Y, Mori S, Igarashi R, Sugi T, Yokota H, Ikeda K, et al. Optically Detected Magnetic Resonance of Nanodiamonds In Vivo; Implementation of Selective Imaging and Fast Sampling. J Nanosci Nanotechnol. 2015 Feb 1;15(2):1014-21.

29. Fu C-C, Lee H-Y, Chen K, Lim T-S, Wu H-Y, Lin P-K, et al. Characterization and application of single fluorescent nanodiamonds as cellular biomarkers. Proc Natl Acad Sci. 2007 Jan $16 ; 104(3): 727-32$.

30. Hui YY, Hsiao WW-W, Haziza S, Simonneau M, Treussart F, Chang H-C. Single particle tracking of fluorescent nanodiamonds in cells and organisms. Curr Opin Solid State Mater Sci. 2017 Feb;21(1):35-42.

31. Liu W, Yu F, Yang J, Xiang B, Xiao P, Wang L. 3D Single-Molecule Imaging of Transmembrane Signaling by Targeting Nanodiamonds. Adv Funct Mater. 2016 Jan;26(3):365-75.

32. McGuinness LP, Yan Y, Stacey A, Simpson DA, Hall LT, Maclaurin D, et al. Quantum measurement and orientation tracking of fluorescent nanodiamonds inside living cells. Nat Nanotechnol. 2011 May 8;6(6):358-63.

33. Kwinter DM, Lo K, Mafi P, Silverman MA. Dynactin regulates bidirectional transport of densecore vesicles in the axon and dendrites of cultured hippocampal neurons. Neuroscience. 2009 Sep;162(4):1001-10.

34. Pinaud F, Clarke S, Sittner A, Dahan M. Probing cellular events, one quantum dot at a time. Nat Methods. 2010 Apr;7(4):275-85.

35. Mudrakola H V, Zhang K, Cui B. Optically Resolving Individual Microtubules in Live Axons. Structure. 2009 Nov 11;17(11):1433-41.

36. Stokin GB, Lillo C, Falzone TL, Brusch RG, Rockenstein E, Mount SL, et al. Axonopathy and transport deficits early in the pathogenesis of Alzheimer's disease. Science. 2005;307(5713):1282-8.

37. Encalada SE, Goldstein LSB. Biophysical Challenges to Axonal Transport: Motor-Cargo Deficiencies and Neurodegeneration. Annu Rev Biophys. 2014 May 6;43(1):141-69.

38. O'Connell MJ, Bachilo SM, Huffman CB, Moore VC, Strano MS, Haroz EH, et al. Band Gap Fluorescence from Individual Single-Walled Carbon Nanotubes. Science. 2002;297(5581):5936.

39. Bachilo SM, Strano MS, Kittrell C, Hauge RH, Smalley RE, Weisman RB. Structure-assigned optical spectra of single-walled carbon nanotubes. Science. 2002;298(5602):2361-6.

40. Tsyboulski DA, Bachilo SM, Weisman RB. Versatile visualization of individual single-walled carbon nanotubes with near-infrared fluorescence microscopy. Nano Lett. 2005;5(5):975-9.

41. Tsyboulski DA, Rocha JDR, Bachilo SM, Cognet L, Weisman RB. Structure-dependent fluorescence efficiencies of individual single-walled carbon nanotubes. Nano Lett. 2007;7(10):3080-5.

42. Cognet L, Tsyboulski DA, Rocha J-DR, Doyle CD, Tour JM, Weisman RB. Stepwise Quenching of Exciton Fluorescence in Carbon Nanotubes by Single-Molecule Reactions. Science. 2007;316(5830):1465-8.

43. Wang F. The Optical Resonances in Carbon Nanotubes Arise from Excitons. Science. 2005;308(5723):838-41.

44. Maultzsch J, Pomraenke R, Reich S, Chang E, Prezzi D, Ruini A, et al. Exciton binding energies in carbon nanotubes from two-photon photoluminescence. Phys Rev B. 2005 Dec 14;72(24):241402.

45. Perebeinos V, Avouris P. Phonon and Electronic Nonradiative Decay Mechanisms of Excitons in Carbon Nanotubes. Phys Rev Lett. 2008 Jul 30;101(5):57401. 
46. Lefebvre J, Austing DG, Bond J, Finnie P. Photoluminescence imaging of suspended singlewalled carbon nanotubes. Nano Lett. 2006;6(8):1603-8.

47. Berciaud S, Cognet L, Lounis B. Luminescence Decay and the Absorption Cross Section of Individual Single-Walled Carbon Nanotubes. Phys Rev Lett. 2008 Aug 14;101(7): 77402.

48. Crochet J, Clemens M, Hertel T. Quantum yield heterogeneities of aqueous single-wall carbon nanotube suspensions. J Am Chem Soc. 2007;129(26):8058-9.

49. Schöppler F, Mann C, Hain TC, Neubauer FM, Privitera G, Bonaccorso F, et al. Molar Extinction Coefficient of Single-Wall Carbon Nanotubes. J Phys Chem C. 2011;115(30):14682-6.

50. Oudjedi L, Parra-Vasquez ANG, Godin AG, Cognet L, Lounis B. Metrological investigation of the $(6,5)$ carbon nanotube absorption cross section. J Phys Chem Lett. 2013;4(9):1460-4.

51. Christofilos D, Blancon JC, Arvanitidis J, Miguel AS, Ayari A, Del Fatti N, et al. Optical imaging and absolute absorption cross section measurement of individual nano-objects on opaque substrates: Single-wall carbon nanotubes on silicon. J Phys Chem Lett. 2012;3(9):1176-81.

52. Vialla F, Roquelet C, Langlois B, Delport G, Santos SM, Deleporte E, et al. Chirality Dependence of the Absorption Cross Section of Carbon Nanotubes. Phys Rev Lett. 2013 Sep 26;111(13):137402.

53. Streit JK, Bachilo SM, Ghosh S, Lin C-W, Weisman RB. Directly Measured Optical Absorption Cross Sections for Structure-Selected Single-Walled Carbon Nanotubes. Nano Lett. 2014;14(3):1530-6.

54. Fakhri N, MacKintosh FC, Lounis B, Cognet L, Pasquali M. Brownian Motion of Stiff Filaments in a Crowded Environment. Science. 2010;330(6012):1804-7.

55. Cherukuri P, Bachilo SM, Litovsky SH, Weisman RB. Near-Infrared Fluorescence Microscopy of Single-Walled Carbon Nanotubes in Phagocytic Cells. J Am Chem Soc. 2004;126(48):15638-9.

56. Reuel NF, Dupont A, Thouvenin O, Lamb DC, Strano MS. Three-dimensional tracking of carbon nanotubes within living cells. ACS Nano. 2012;6(6):5420-8.

57. Fakhri N, Wessel AD, Willms C, Pasquali M, Klopfenstein DR, MacKintosh FC, et al. Highresolution mapping of intracellular fluctuations using carbon nanotubes. Science. 2014 May 30;344(6187):1031-5.

58. Cambré S, Santos SM, Wenseleers W, Nugraha ART, Saito R, Cognet L, et al. Luminescence properties of individual empty and water-filled single-walled carbon nanotubes. ACS Nano. 2012;6(3):2649-55.

59. Duque JG, Oudjedi L, Crochet JJ, Tretiak S, Lounis B, Doorn SK, et al. Mechanism of electrolyteinduced brightening in single-wall carbon nanotubes. J Am Chem Soc. 2013;135(9):3379-82.

60. Heller DA, Jin H, Martinez BM, Patel D, Miller BM, Yeung TK, et al. Multimodal optical sensing and analyte specificity using single-walled carbon nanotubes. Nat Nanotechnol. 2009;4(2):114-20.

61. Jin H, Heller DA, Kalbacova M, Kim J-H, Zhang J, Boghossian AA, et al. Detection of singlemolecule $\mathrm{H} 2 \mathrm{O} 2$ signalling from epidermal growth factor receptor using fluorescent singlewalled carbon nanotubes. Nat Nanotechnol. 2010;5(4):302-9.

62. Heller DA, Pratt GW, Zhang J, Nair N, Hansborough AJ, Boghossian AA, et al. Peptide secondary structure modulates single-walled carbon nanotube fluorescence as a chaperone sensor for nitroaromatics. Proc Natl Acad Sci. 2011;108(21):8544-9.

63. Kruss S, Landry MP, Vander Ende E, Lima BMA, Reuel NF, Zhang J, et al. Neurotransmitter detection using corona phase molecular recognition on fluorescent single-walled carbon nanotube sensors. J Am Chem Soc. 2014;136(2):713-24.

64. Budhathoki-Uprety J, Langenbacher RE, Jena P V., Roxbury D, Heller DA. A Carbon Nanotube Optical Sensor Reports Nuclear Entry via a Noncanonical Pathway. ACS Nano.

2017;11(4):3875-82.

65. Ju SY, Kopcha WP, Papadimitrakopoulos F. Brightly fluorescent single-walled carbon nanotubes via an oxygen-excluding surfactant organization. Science. 2009;323(5919):131923. 
66. Diao S, Hong G, Antaris AL, Blackburn JL, Cheng K, Cheng Z, et al. Biological imaging without autofluorescence in the second near-infrared region. Nano Res. 2015;8(9):3027-34.

67. Hong G, Diao S, Antaris AL, Dai H. Carbon Nanomaterials for Biological Imaging and Nanomedicinal Therapy. Chem Rev. 2015 Oct 14;115(19):10816-906.

68. Welsher K, Liu Z, Sherlock SP, Robinson JT, Chen Z, Daranciang D, et al. A route to brightly fluorescent carbon nanotubes for near-infrared imaging in mice. Nat Nanotechnol. 2009;4(11):773-80.

69. Hong G, Diao S, Chang J, Antaris AL, Chen C, Zhang B, et al. Through-skull fluorescence imaging of the brain in a new near-infrared window. Nat Photonics. 2014;8(9):723-30.

70. Berg HC. Random Walks in Biology. Princeton University Press; 1993.

71. Dusenbery DB. Living at Micro Scale: The Unexpected Physics of Being Small. Harvard University Press; 2009.

72. Thompson RE, Larson DR, Webb WW. Precise Nanometer Localization Analysis for Individual Fluorescent Probes. Biophys J. 2002 May;82(5):2775-83.

73. Yildiz A. Myosin V Walks Hand-Over-Hand: Single Fluorophore Imaging with 1.5-nm Localization. Science. 2003 Jun 27;300(5628):2061-5.

74. Biermann B, Sokoll S, Klueva J, Missler M, Wiegert JS, Sibarita J-B, et al. Imaging of molecular surface dynamics in brain slices using single-particle tracking. Nat Commun. 2014 Jan 16;5:3024.

75. Varela JA, Dupuis JP, Etchepare L, Espana A, Cognet L, Groc L. Targeting neurotransmitter receptors with nanoparticles in vivo allows single-molecule tracking in acute brain slices. Nat Commun. 2016 Mar 14;7:10947.

76. Wenseleers W, Vlasov IL, Goovaerts E, Obraztsova ED, Lobach AS, Bouwen A. Efficient isolation and solubilization of pristine single-walled nanotubes in bile salt micelles. Adv Funct Mater. 2004;14(11):1105-12.

77. Duque JG, Pasquali M, Cognet L, Lounis B. Environmental and synthesis-dependent luminescence properties of individual single-walled carbon nanotubes. ACS Nano. 2009;3(8):2153-6.

78. Gao Z, Danné N, Godin A, Lounis B, Cognet L. Evaluation of Different Single-Walled Carbon Nanotube Surface Coatings for Single-Particle Tracking Applications in Biological Environments. Nanomaterials. 2017 Nov 16;7(11):393.

79. Bisker G, Dong J, Park HD, Iverson NM, Ahn J, Nelson JT, et al. Protein-targeted corona phase molecular recognition. Nat Commun. 2016 Jan 8;7:10241.

80. Danné N, Godin AG, Gao Z, Varela JA, Groc L, Lounis B, et al. Comparative Analysis of Photoluminescence and Upconversion Emission from Individual Carbon Nanotubes for Bioimaging Applications. ACS Photonics. 2017 Dec 8; acsphotonics.7b01311.

81. Fakhri N, Tsyboulski DA, Cognet L, Weisman RB, Pasquali M. Diameter-dependent bending dynamics of single-walled carbon nanotubes in liquids. Proc Natl Acad Sci. 2009 Aug 25;106(34):14219-23.

82. Odijk T. On the Statistics and Dynamics of Confined or Entangled Stiff Polymers. Macromolecules. 1983;16(8):1340-4.

83. de Gennes PG. Reptation of a Polymer Chain in the Presence of Fixed Obstacles. J Chem Phys. 1971;55(2):572-9.

84. Doi M. Rotational relaxation time of rigid rod-like macromolecule in concentrated solution. J Phys. 1975;36(7-8):607-11.

85. Jena P V., Shamay Y, Shah J, Roxbury D, Paknejad N, Heller DA. Photoluminescent carbon nanotubes interrogate the permeability of multicellular tumor spheroids. Carbon N Y. 2016 Feb;97:99-109.

86. Wang Y, Bahng JH, Che Q, Han J, Kotov NA. Anomalously Fast Diffusion of Targeted Carbon Nanotubes in Cellular Spheroids. ACS Nano. 2015;9(8):8231-8.

87. Korogod N, Petersen CC, Knott GW. Ultrastructural analysis of adult mouse neocortex 
comparing aldehyde perfusion with cryo fixation. Elife. 2015 Aug 11;4:e05793.

88. Mohan N, Tzeng Y-K, Yang L, Chen Y, Hui YY, Fang C-Y, et al. Sub-20-nm Fluorescent Nanodiamonds as Photostable Biolabels and Fluorescence Resonance Energy Transfer Donors. Adv Mater. 2010 Feb 16;22(7):843-7.

89. Boudou J-P, Tisler J, Reuter R, Thorel A, Curmi PA, Jelezko F, et al. Fluorescent nanodiamonds derived from HPHT with a size of less than 10nm. Diam Relat Mater. 2013 Aug;37:80-6.

90. Su L-J, Wu M-S, Hui YY, Chang B-M, Pan L, Hsu P-C, et al. Fluorescent nanodiamonds enable quantitative tracking of human mesenchymal stem cells in miniature pigs. Sci Rep. 2017 Mar 30;7:45607.

91. Alhaddad A, Adam M-P, Botsoa J, Dantelle G, Perruchas S, Gacoin T, et al. Nanodiamond as a Vector for siRNA Delivery to Ewing Sarcoma Cells. Small. 2011 Nov 4;7(21):3087-95.

92. Slegerova J, Hajek M, Rehor I, Sedlak F, Stursa J, Hruby M, et al. Designing the nanobiointerface of fluorescent nanodiamonds: highly selective targeting of glioma cancer cells. Nanoscale. 2015;7(2):415-20. 


\section{FIGURES}

(a)

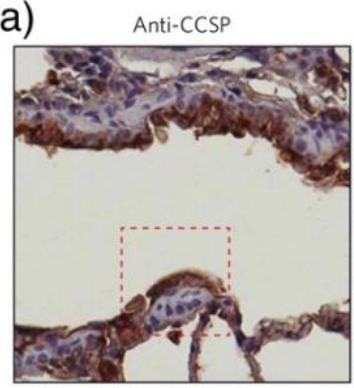

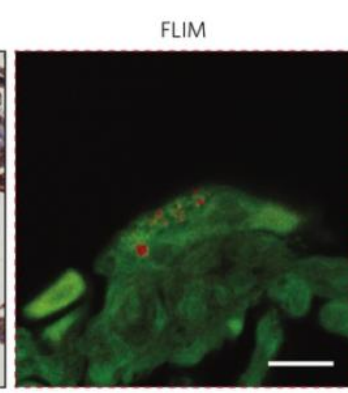

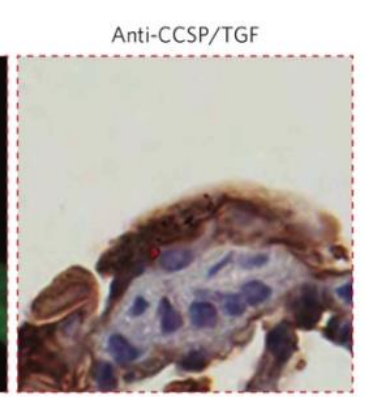

(b)

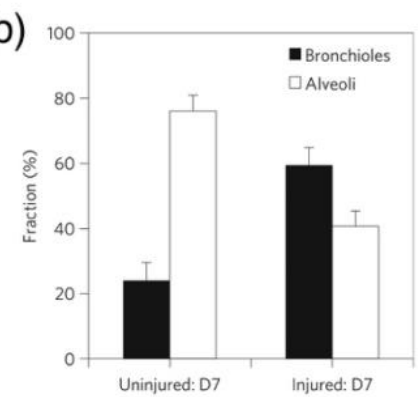

Figure 1 - Identification of transplanted FND-labelled lung stem cells in bronchiolar epithelia and their histomorphometric distribution. (a), Lung tissue sections prepared from naphthalene-injured mice on day 7 after intravenous injection of FND-labelled LSCs. (b) left: immunostaining (brown) of club cells with via the targeting of CCSP (club cell secretory protein) marker. The enlarged images in the red dashed boxes show FLIM (red color correspond to $\approx 20$ ns fluorescence lifetime, and green to $\approx 2 \mathrm{~ns}$ ) in the middle, and (a), right: merge of anti-CCSP and time-gating fluorescence (red) signals. FNDlabelled LSCs (red) co-localize with CCSP $^{+}$(brown) cells and reside at the bronchiolar epithelial region of the lung. Scale bar: $10 \mu \mathrm{m}$. (b) Histomorphometric distribution measured by visually counting the numbers of FND-labelled LSCs nested in terminal bronchioles (black) or alveoli (white) of the lungs of uninjured and injured mice on day 7 after intravenous injection. Results are averages of more than 30 visual fields per section, with error bars representing standard deviations of the measurements ( $n=6$ per group). Adapted from Ref. (18) with permission. Copyright 2013 Springer Nature Group. 

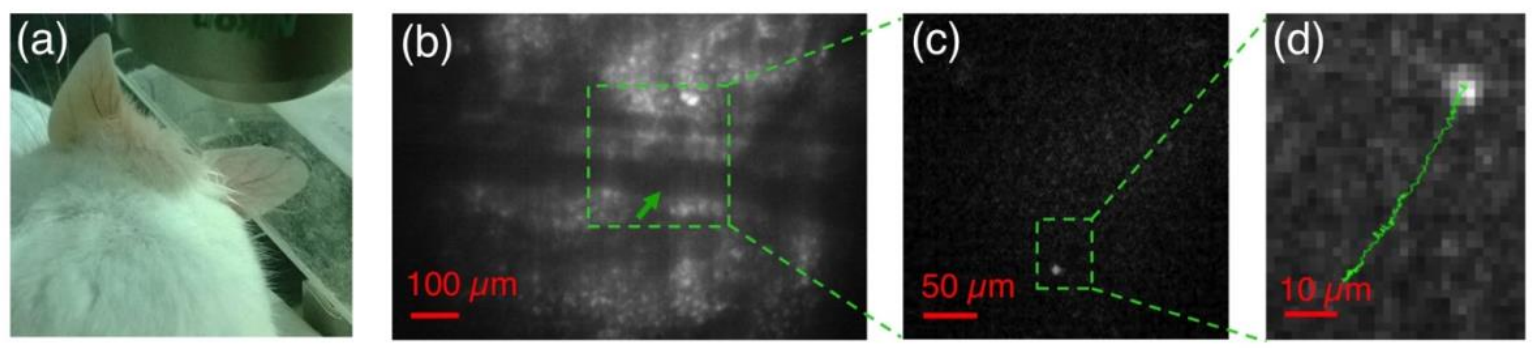

Figure 2 - In vivo imaging and tracking of FND-labeled cancer cells in a mouse ear blood vessels, after intravenous injection. (a) Photograph of the experimental arrangement. (b) Bright-field image of a mouse ear tissue (no time-gating). The green arrow indicates the position of an FND-labeled lung cancer cell in the blood vessel of $\approx 50 \mu \mathrm{m}$ in diameter. (c) Enlarged view of (b) fluorescence image, in time-gated mode, and after subtraction of stationary autofluorescence background, showing one bright spot which corresponds to a FND-labeled lung cancer cell. (d) Enlarged view of the time-gated fluorescence image (rectangular green region in (c)), showing the trajectory of the FND-labeled cell in the vessel. Adapted from Ref. (23) with permission. Copyright 2014 Springer Nature Group. 
(a1)

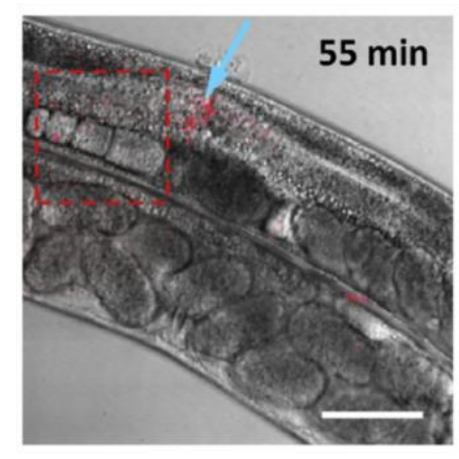

(b1)

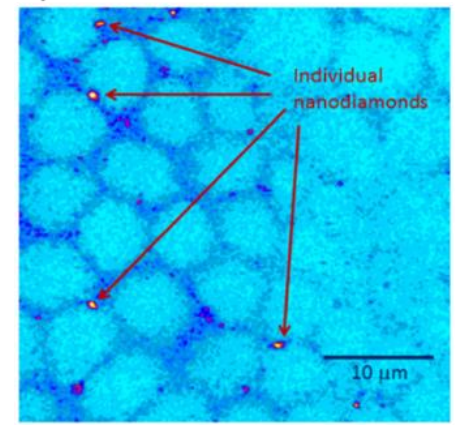

(a2)
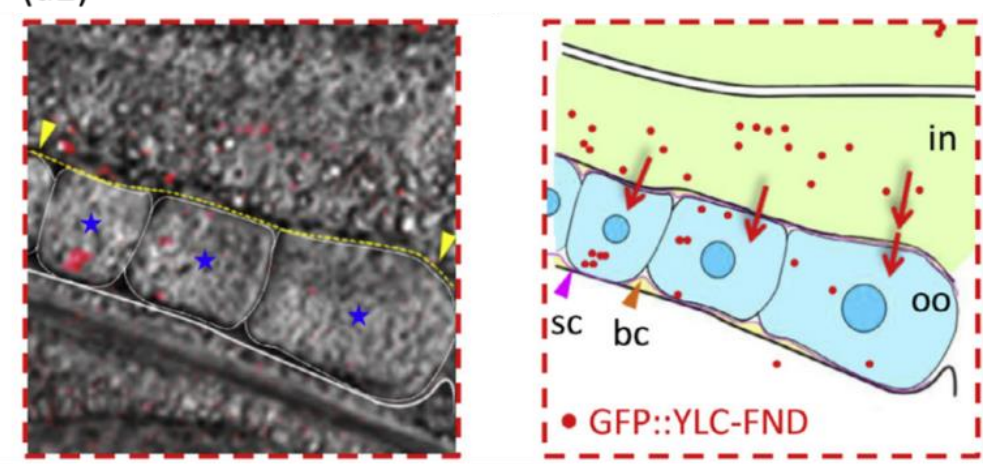

(b2)

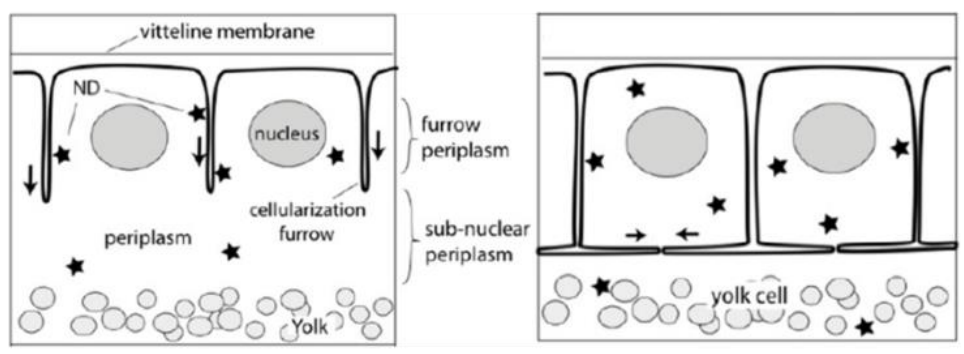

Figure 3 - Tracking protein conjugated-FND in C-elegans worm and drosophila embryo. (a1)

Superposition of bright-field and time-gated fluorescence image of a representative $C$-elegans worm at 55 min after injection of GFP::YLC-FNDs into the intestinal cell. The blue arrow indicates the injection site. Scale bars: $50 \mu \mathrm{m}$. (a2) An enlarged image (left) and a cartoon (right) of the area in the red box in (a1), showing the presence of GFP::YLC-FNDs in both intestine (left: yellow dotted line for the boundary; right: 'in') and oocytes (left: thin white lines and nuclei labeled with blue stars; right: 'oo'). Adapted from Ref. (25) with permission. Copyright 2013 Elsevier. (b1) Scanning confocal fluorescence image of individual nanodiamonds in the blastoderm cells during stage 5 of development of a drosophila embryo. The image shows the auto-fluorescence from the junction of each blastoderm cell as well as the strong fluorescent signal from individual nanodiamonds which in the majority of cases is localized to the cell periphery. (b2) Schematic of the micro-injection of nanodiamonds into the drosophila embryo. Embryos at early (left) and late (c) stage 5 showing the cellularization furrows introgressing between nuclei, which invade the yolk-free periplasm during the later syncytial divisions (right, arrows). The ingressing membranes eventually join and pinch off individual blastoderm cells, forming a large, internal yolk cell. Nanodiamonds that have diffused into the yolk-free periplasm can become internalized in the blastoderm cells at the completion of stage 5. Adapted from Ref. (26) with permission. Copyright 2014 Optical Society of America. 
(a)
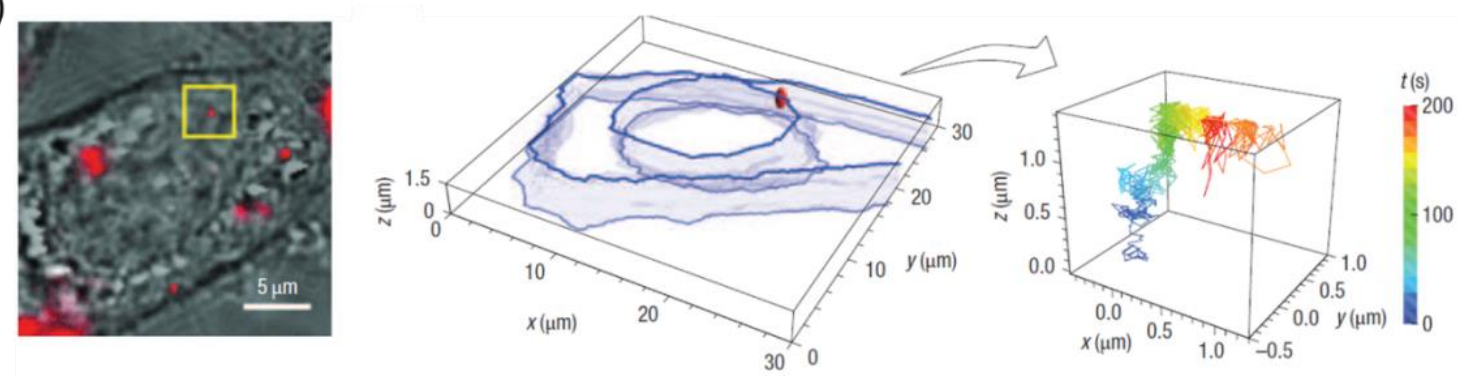

(b1)

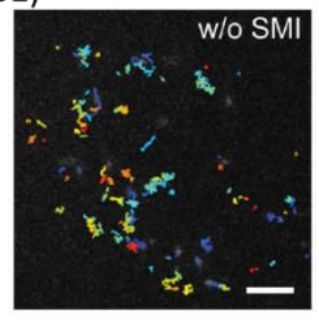

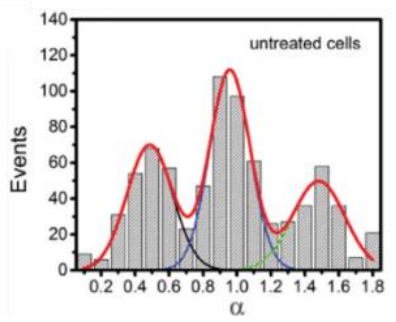

(b2)
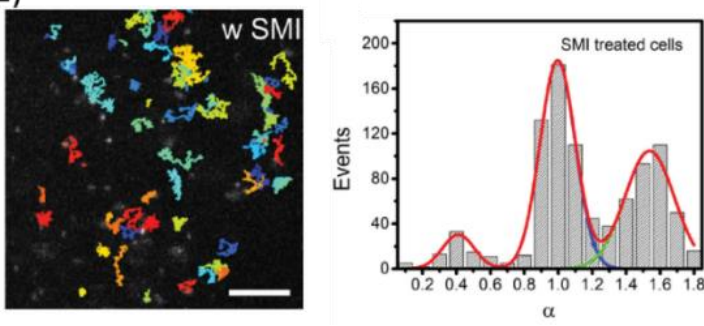

Figure 4 - Intracellular tracking of single FNDs, one cell at a time. (a) 3D-tracking of a single 35-nm FND in a live HeLa cell. Left: overlay of bright-field and red epifluorescence images of the cell after FND uptake. Middle: 3D-reconstruction showing the nucleus and cytoplasm of the cell, as well as the red spot of the internalized FND. Right: 3D-trajectory reconstruction of a single FND (surrounded by a yellow box in a) inside the cell over a time span of $200 \mathrm{~s}$. Adapted from Ref. (12) with permission. Copyright 2008 Springer Nature Group. (b) Single particle tracking of TGF- $\beta$ membrane receptors labeled with TGF-conjugated FND in living cancer cell line. (b1) Left: TGF- $\beta$ trajectories overlaid with wide-field fluorescence images without SMI treatment. Color corresponds to the track numbers. Scale bar: $3 \mu \mathrm{m}$. Right: distribution of the anomalous diffusion parameter $\alpha$, showing three prominent peaks corresponding to the three different diffusion regimes (peaks from left to right: immobile, anomalous and Brownian). (b2) Same as (b1) but with SMI treatment of cancer cell culture. Note that the fraction of immobile particles decreases substantially. Adapted from Ref. (31) with permission. Copyright 2016 Wiley-VCH. 
(a)

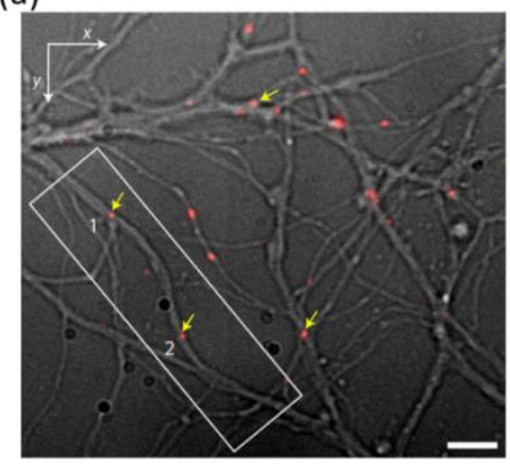

(b)

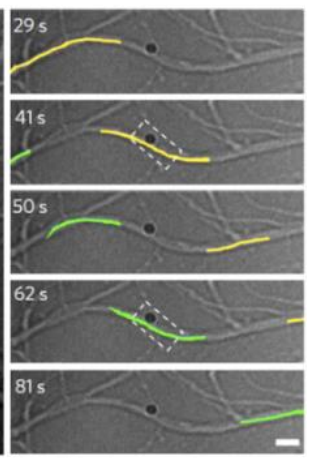

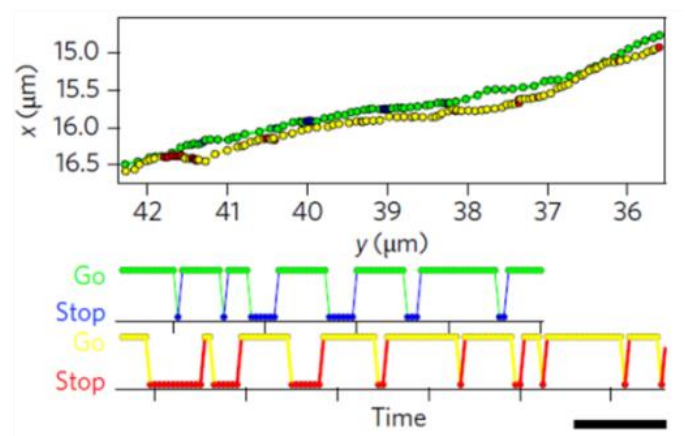

(c)
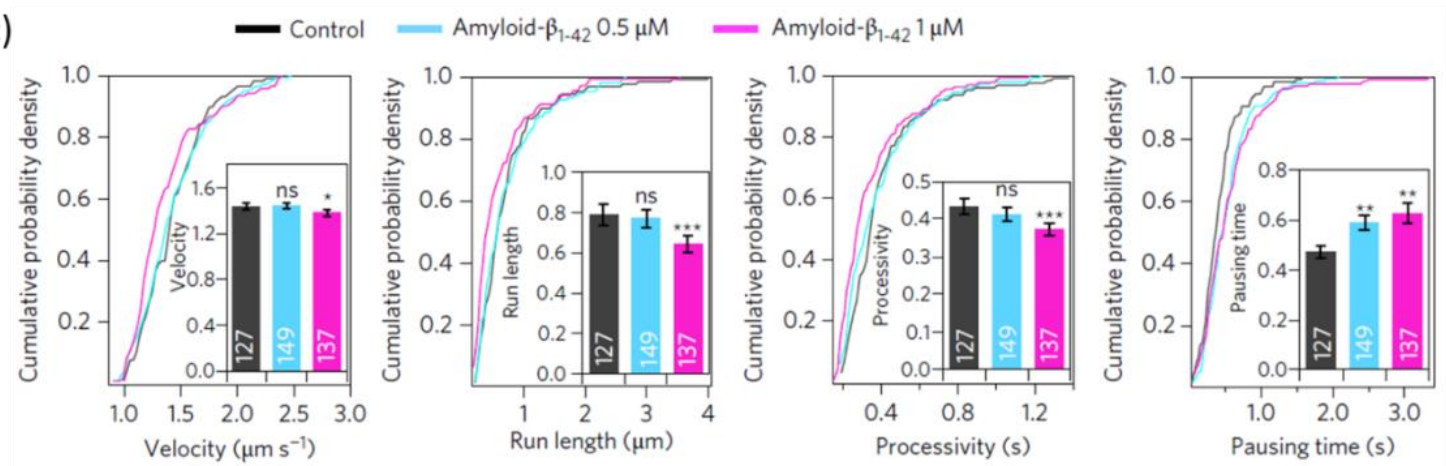

Figure 5 - Single FND tracking inside branches of neuron reveals intraneuronal transport abnormalities. (a) Left: bright-field image merged with the FND red channel showing four fNDs moving inside neuronal branches (yellow arrows). The FND-containing endosomes labeled \#1 and \#2 (solid white line box) were observed moving in the same branch and towards the cell soma (not visible in this image). Scale bars: $5 \mu \mathrm{m}$. Right: snapshot at different time of the two FNDs emphasizing their trajectory (yellow and green respectively; from white box in (a), left). Scale bars: $1 \mu \mathrm{m}$. (b) Top: trajectories of FND \#1 and \#2 extracted from dashed line rectangle in (a, right). The two colors in each trajectory correspond to "go" phases (in green for FND \#1 and yellow for FND \#2) and "stop" phases (in blue for FND \#1 and red for FND \#2). Bottom: two-state ("stop" and "go") representation of the same trajectories over time: FND \#1 moved faster than FND \#2 (scale bar: $1 \mathrm{~s}$ ). (c) application of the FND-tracking assay to Alzheimer's disease model. $A \beta_{1-42}$ used at concentrations of $0.5 \mu \mathrm{M}$ (blue) and $1 \mu \mathrm{M}$ (purple) induce an increase in pausing time concentration as small as $0.5 \mu \mathrm{M}$ and significant changes of all parameters at $1 \mu \mathrm{M}$. Figures written inside the bars represent the total number of trajectories. Data are displayed as cumulative probabilities and bar plots with mean values \pm s.e.m in insets. Statistical significance: one star $\left({ }^{*}\right)$ indicate a $p$ value smaller than 0.05 (Wilcoxon and MannWhitney two-tailed test), two stars $\left({ }^{* *}\right)$ indicate a $p<0.01$ and three stars $\left({ }^{* * *}\right)$ mean that $p<0.001$. Adapted from Ref. (1) with permission. Copyright 2017 Springer Nature Group. 


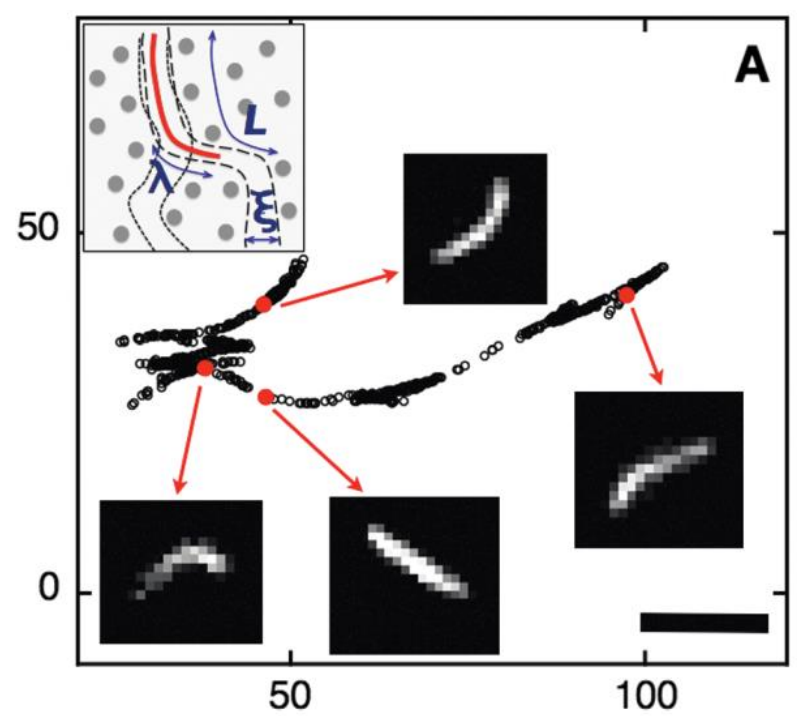

Figure 6 - SWCNT reptation dynamics revealed in a complex biocompatible environment, under a more than 20 min continuous imaging. Adapted from Ref. (54). Copyright 2010 Science.

(a)

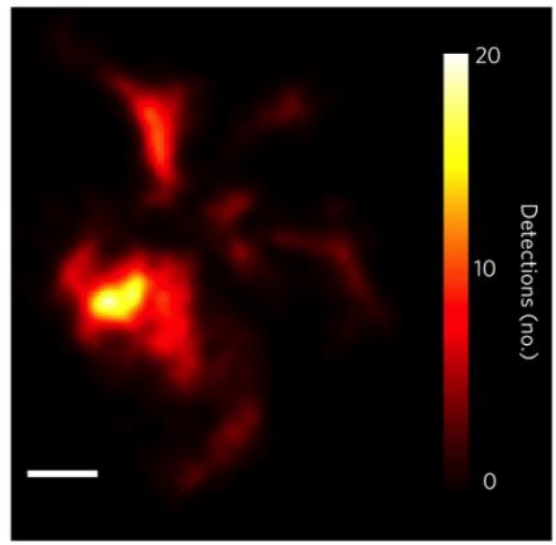

(b)

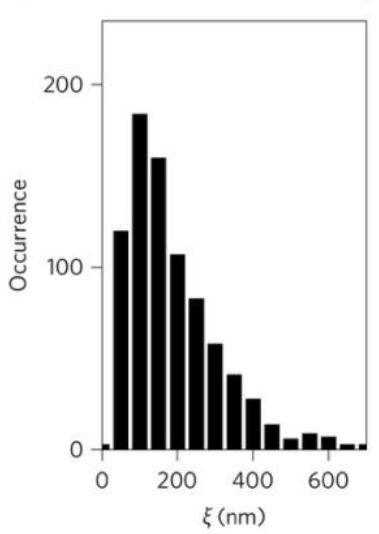

(c)

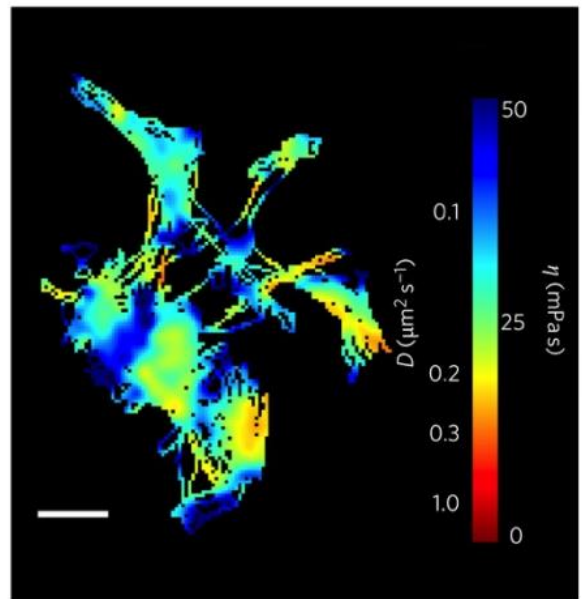

Figure 7 - Super-resolved map (a) and viscosity map (c) of the extracellular space obtained in acute rat brain slices from the analysis of single SWCNT diffusion. In the central panel (b), the dimensions of the extracellular space $\xi$ could be resolved at resolution smaller than the diffraction limit. Adapted from Ref. (2). Copyright 2017 Springer Nature Group. 